\title{
Hepatitis E Virus Infection in a Student Healthcare Worker: A Case Report
}

\author{
Antoon De Schryver ${ }^{1,2 *}$, Inge Wijnants ${ }^{2}$, Paolo Bonanni ${ }^{3}$, Ramona Hambach ${ }^{1}$, Marc van Sprundel ${ }^{1}$ \\ and Guido François ${ }^{1}$
}

\author{
${ }^{1}$ Department of Epidemiology and Social Medicine, University of Antwerp, Antwerp, Belgium \\ ${ }^{2}$ IDEWE Occupational Health Services, Leuven, Belgium \\ ${ }^{3}$ Department of Health Sciences, University of Florence, Florence, Italy
}

*Corresponding author: Antoon De Schryver, Department of Epidemiology and Social Medicine, Campus Drie Eiken, University of Antwerp, Universiteitsplein 1, BE-2610 Antwerp, Belgium, Tel: +32-3-265-25-24, Fax: +32-3-265-28-75, E-mail: antoon.deschryver@uantwerpen.be

\begin{abstract}
Against the background of hepatitis prevention and control in Belgium, an important focus is on healthcare and occupational medicine settings. Most Belgian healthcare workers in training are administered a combined hepatitis $A / B$ vaccine by occupational health services.

A female healthcare trainee presented herself to her General Practitioner (GP) with general fatigue after receipt of the first two doses $(0,1,6$ months scheme) of her vaccination course. Serum Aspartate Aminotransferase (AST), Alanine Aminotransferase (ALT), Gamma-Glutamyl Transferase (GGT), and Alkaline Phosphatase (ALP) concentrations were severely elevated, while Total Bilirubin (TBIL) and Lactate Dehydrogenase (LDH) levels both fell within the normal range. The patient was Anti-HAV Immunoglobulin M (IgM) and Immunoglobulin $\mathrm{G}(\mathrm{IgG})$ positive, and HBV surface Antigen ( $\mathrm{HBsAg}$ ) and Anti-HBV core antigen (Anti-HBc) negative. Her serum Anti-HBs concentration mounted to 302 IU/l. Anti-HCV antibody concentrations were below the detection limit. It was originally assumed that the patient was HAV-infected due to hepatitis A vaccine failure. Additional blood examination, performed before the third vaccine dose was administered, revealed the occurrence of Anti-HEV IgM and IgG. Consensus was reached that the initial Anti-HAV IgM and IgG levels were due to vaccination, while a coinciding HEV infection resulted in fatigue, high ALT and AST levels, and Anti-HEV IgM and IgG positivity.

$\mathrm{HEV}$ is the most common cause of acute hepatitis worldwide. Since autochthonous HEV infection is becoming more frequent and can easily be overlooked in occupational medicine and general practices, serological testing for hepatitis E deserves special attention in comparable circumstances.
\end{abstract}

\author{
Keywords \\ Hepatitis $A$ and $E$ virus infection, Combined hepatitis $A / B$ \\ vaccination, Elevated aminotransferase levels and fatigue, \\ Occupational setting
}

\begin{abstract}
Abbreviations
GP: General Practitioner; AST: Aspartate Aminotransferase; ALT: Alanine Aminotransferase; GGT: Gamma-Glutamyl Transferase; ALP: Alkaline Phosphatase; TBIL: Total Bilirubin; LDH: Lactate Dehydrogenase; HAV: Hepatitis A Virus; HBV: Hepatitis B Virus; HCV: Hepatitis C Virus; HEV: Hepatitis E Virus; Anti-HAV IgM: IgM Antibody to HAV; Anti-HAV IgG: IgG Antibody to HAV; HBsAg: HBV surface Antigen; Anti-HBs: Antibody to HBsAg; Anti-HBc: Antibody to HBV core antigen; Anti-HCV: Antibody to HCV; Anti-HEV IgM: IgM Antibody to HEV; Anti-HEV IgG: IgG Antibody to HEV
\end{abstract}

\section{Introduction}

Hepatitis E Virus (HEV) is a small non-enveloped virus with a positive-sense, single-stranded $7.2 \mathrm{~kb}$ RNA genome, first recognized in the early 1980s [1]. HEV belongs to the Hepeviridae, a family consisting of two genera: Pescihepevirus, infecting trout, and Orthohepevirus, infecting mammals and avians. Viral variants infecting humans belong to the species Orthohepevirus $A$, a member of the genus Orthohepevirus. There are currently eight Orthohepevirus A genotypes identified $[2,3]$. 
HEV is the most common cause of acute hepatitis worldwide. Every year there are an estimated 20 million HEV infections, over 3 million symptomatic cases, and 60,000 HEV-related deaths $[4,5]$. HEV is endemic in many developing countries where it causes substantial morbidity. In these countries, the virus, particularly genotypes 1 and 2, is transmitted primarily by the faeco-oral route and is associated with both sporadic infections and epidemics in areas of poor sanitation and weak public health infrastructures. In industrialized countries HEV infections were traditionally thought to occur only in individuals who had become infected when travelling in areas where the virus is endemic.

Zoonotic spread of the virus is considered a possibility for genotypes 3 and 4 , since different groups of mammals, such as pigs, cows, sheep, goats, rodents, and non-human primates have been shown to be susceptible to infection. Genotype 3 is mostly present in industrialized countries, and genotype 4 in Asian countries [6]. Person-to-person transmission of any type is probably uncommon [5]. It has been estimated that at least one third of the world population, mainly persons living in Africa, Asia, Central America, and the Middle East has ever been infected.

Genotypes 5 and 6 infect wild boars, and genotypes 7 and 8 infect camels [7]. Genotype 7 has been isolated from a solid organ transplant patient who consumed camel meat and milk [8].

HEV infection is rarely detected in young children. Young- to middle-aged adults appear to be most susceptible, and antibody prevalence increases with age. As for the clinical importance of HEV infection, it must be pointed out that most HEV-infected people recover completely. The overall case fatality rate is about $1 \%$. However, several population groups are at increased risk.

Firstly, HEV genotypes 1 and 2 are highly dangerous for pregnant women, who are at risk of serious illness and mortality. In the third trimester of pregnancy, mortality rates can be as high as $10-30 \%$. It is not yet clear if genotypes 3 and 4 have the same impact on pregnant women, but European studies [9] suggest that this is not the case. Secondly, HEV infection potentially leads to decompensation and mortality in people with pre-existing chronic liver disease. Thirdly, HEV infection has been shown to be associated with a range of neurological syndromes including Guillain-Barré [4]. And fourthly, in contrast to the situation in developing countries, an increasing number of cases progressing to chronic hepatitis and chronic liver disease are being reported in developed countries. Those at risk are mainly people on immunosuppressive treatment following solid organ transplants. So, if HEV is occupationally transmitted, this would have some serious consequences for different groups of workers [6].
Data from studies conducted during the past decade have greatly shifted our knowledge on the epidemiology of HEV. Recently it has been shown that contrary to previous beliefs, hepatitis $E$ is also an endemic disease in several developed countries [10-12] as evidenced by reports of high Anti-HEV IgG levels in healthy individuals and an increasing number of non-travel related HEV cases [13]. Moreover, a porcine reservoir and growing evidence of zoonotic transmission have been reported in these countries [14]. Accumulating evidence surrounding cases of HEV infection in these countries suggest that they are due to autochthonous (i.e. locally acquired) viral strains, nearly always classified as viral genotype 3 . In a recent study of around 3500 subjects with suspected hepatitis from Scotland, the frequency of detection of HEV was over 31 times higher than that of HAV, confirming that HEV is an increasingly important cause of acute hepatitis [15]. However, in these low-endemicity areas, studies have shown that men are more frequently positive than women, and particularly that those in animal care occupations, such as pig breeders, veterinarians, or slaughterhouse personnel, are statistically more frequently positive for Anti-HEV antibodies [16]. The true global burden of hepatitis $E$ disease is unknown and many aspects of the epidemiology in, e.g., European countries remain to be elucidated $[17,18]$. This is particularly so for the possibility of HEV being an occupational infection in pig workers [6].

Against the background of hepatitis prevention and control in Belgium, an important focus is on healthcare and occupational medicine settings. Hepatitis $B$ vaccination for Belgian Healthcare Workers (HCWs), including those in training, is mandatory since 1999. Initially, a monovalent vaccine used to be given in a $0,1,2,12$ months scheme before or shortly after a trainee started his or her education. Later on, the hepatitis B vaccine was in the majority of cases replaced by a combined hepatitis $A / B$ vaccine, administered in a 0, 1, 6 months scheme.

\section{Methods and Results}

A female first-year student nurse, aged 48 , went to see her General Practitioner (GP) on March 15 of her first year, with complaints of general fatigue, but no other, specific symptoms of disease. She reported no history of previous or current contact with animals (particularly pigs), no contact with water during recent flooding or no recent travel in a developing country. An occupational physician had administered her first and second doses of hepatitis A/B vaccine (Twinrix Adult ${ }^{\circ}$, GlaxoSmithKline Biologicals, Rixensart, Belgium), 3 and 2 months before, (on December 17 and January 26, resp). Her GP ordered a blood examination, which yielded a number of abnormal liver values (normal range between brackets), including Aspartate Aminotransferase (AST): $243 \mathrm{mU} / \mathrm{ml}(0-30 \mathrm{mU} / \mathrm{ml})$; Alanine Aminotransferase (ALT): $535 \mathrm{mU} / \mathrm{ml}(0-30 \mathrm{mU} / \mathrm{ml})$; Gamma-Glutamyl 
Table 1: Summary of patient data and test results.

Patient: Female first-year student nurse, aged 48

Month 1 First hepatitis A/B vaccine dose administered

Month 2 Second hepatitis A/B vaccine dose administered

Month 3 First examination (GP setting), yielding:

1) Clinical symptoms: General fatigue.

2) Elevated liver enzyme values (AST, ALT, GGT, and ALP).

3) HAV, HBV, and HCV serology: Anti-HAV IgM and Anti-HAV IgG: Positive; HBsAg: Negative; Anti-HBs: 302 IU/l; Anti-HBc and Anti-HCV: Negative.

Month 6 Second examination (occupational medicine setting), yielding:

1) Clinical symptoms: Resolved.

2) Normal liver enzyme values (AST, ALT, GGT, and ALP).

3) HAV, HBV, and HCV serology: Anti-HAV IgM: Negative; Anti-HAV IgG: Positive; HBsAg: Negative; Anti-HBs: $505 \mathrm{IU} / \mathrm{l} ;$ Anti-HBc and Anti-HCV: Negative.

4) HEV serology: Anti-HEV IgM and Anti-HEV IgG: Positive.

Month 6 Third hepatitis A/B vaccine dose administered

Transferase (GGT): $240 \mathrm{mU} / \mathrm{ml}(0-38 \mathrm{mU} / \mathrm{ml}) ;$ and Alkaline Phosphatase (ALP): $593 \mathrm{mU} / \mathrm{ml}(64-300 \mathrm{mU} / \mathrm{ml})$. Serum concentrations of Total Bilirubin (TBIL) and Lactate Dehydrogenase (LDH) both fell within the normal range. Serological results for Hepatitis A Virus (HAV), Hepatitis $B$ Virus (HBV), and Hepatitis C Virus (HCV) included: Anti-HAV IgM (IgM Antibody to HAV): Positive; Anti-HAV IgG (IgG Antibody to HAV): Positive; HBsAg (HBV surface Antigen): Negative; Anti-HBs (Antibody to HBsAg): 302 IU/I; Anti-HBC (Antibody to HBV core antigen): Negative; and Anti-HCV (Antibody to HCV): Negative (Table 1).

An additional blood examination was performed as ordered by the occupational physician at the time the third hepatitis $A / B$ vaccine dose was planned to be administered, i.e. at month 6 (June 16). The liver enzyme values AST, ALT, GGT, ALP had all dropped below the Upper Limits of Normal (ULNs). The serological results were as follows: Anti-HAV IgM: Negative; Anti-HAV IgG: Positive; HBsAg: Negative; Anti-HBs: 505 IU/I; Anti-HBc: Negative; and Anti-HCV: Negative. In contrast with the first examination, also HEV serological parameters were determined. Both Anti-HEV IgM (IgM Antibody to HEV) and Anti-HEV IgG (IgG Antibody to HEV) levels proved to be positive (Table 1). Tests were performed by the Belgian National Reference Centre for Measles, Mumps, Rubella, and Viral Hepatitis. A first screening for Anti-HEV IgM and IgG was done with IgM/IgG recom Well ELISA (Mikrogen GmbH, Neuried, Germany). Positive results were confirmed with the immunoblot recomblot IgM/IgG (Mikrogen GmbH, Neuried, Germany).

At the time the trainee received her third vaccination dose (month 6), she showed no longer any clinical symptoms and had apparently recovered very well from her infection.

\section{Discussion}

Severely elevated levels of AST, ALT, GGT, and ALP point to liver damage, possibly due to a viral infection. HBV infection could be excluded in this case, since at the two mentioned occasions HBsAg was negative, An-
ti-HBs levels were highly above the protection threshold (10 IU/I), and Anti-HBc was negative. This combination of results indicates immunity against hepatitis $B$ due to vaccination [19]. The patient was not currently nor previously HCV infected either, since Anti-HCV was and remained negative.

At first sight, HAV infection could be ruled out as well, since the student had received two vaccine doses and primary HAV vaccine failure is very rare $[20,21]$. The combined results of the clinical and blood examination (fatigue, elevated liver enzyme values, Anti-HAV IgM and IgG positivity), however, seemed to contradict this assumption. On the other hand, both Anti-HAV IgM and IgG positivity can indeed be linked to vaccination only. Vaccine-induced Anti-HAV IgM has been described by several authors, in varying proportions of vaccinees and up to several months after the primary course was completed [21], while achieving high IgG levels is one of the main objectives of vaccination. Also, the timing of Anti-HAV IgG appearance is normal in the case under consideration, since it has been shown that full protection against $\mathrm{HAV}$ infection is generally reached after the second vaccine dose [21].

The student's reported fatigue could have had a multitude of causes. But, if vaccination is sufficient to explain the appearance of Anti-HAV IgM and IgG, what was then the cause of the severely elevated liver values? There is no way to explain them as a consequence of vaccination; there are described examples of increased ALT and AST levels in healthy individuals after HAV vaccination, but the elevations are qualified as slight, e.g., 1.5 times above the ULN, and transient (back to normal three months after vaccination) [22]. It is clear that the levels measured on March 15 (18 and 8 times the ULN, for ALT and AST, resp), were much too high to fit within this scheme. Hence, at that moment, the provisional diagnosis was HAV infection and hepatitis A vaccine failure, a rare condition that has been documented [20,21].

Serious liver damage can, except by viral hepatitis, also be caused by a large number of drugs and substanc- 
es; examples are acetaminophen taken in overdoses, fluoxetine (e.g., Prozac ${ }^{\circledR}$, Eli Lilly and Company, Indianapolis, USA), statins [23], diclofenac (e.g., Voltaren ${ }^{\circledR}$, Novartis International AG, Basel, Switzerland) in $0.5 \%$ of all cases [24], and certain herbal remedies. Finally, a rather small proportion of patients on Highly Active Antiretroviral Therapy (HAART) show signs of hepatotoxicity, illustrated by ALT elevations more than 10 times the ULN [25]. The anamnesis of our patient, and the further clinical course, however, did not point to any of the potential causes of liver injury listed above. Alcohol abuse could probably be ruled out, since we found an AST/ALT ratio of about 0.45 , while a ratio greater than 2 is considered characteristic in alcoholic hepatitis [26].

The second series of observations (June 16) changed the whole picture. The originally measured Anti-HAV IgM level had turned negative over time, as could have been expected and confirming that it had been caused by vaccination. Most importantly, however, both the observed Anti-HEV IgM and IgG levels are consistent with HEV infection. Infection probably occurred shortly before the first blood examination and explains the fatigue and the severely elevated liver enzyme values measured at that time. And although Anti-HEV IgM and IgG levels were not measured in parallel, it is rather safe to assume that both of them had been positive in the first series of observations as well.

Some caution is generally needed regarding the performance of Anti-HEV assays. It has been shown by others [27] that frequently used tests for HEV detection show clear differences in diagnostic sensitivity. That is the reason why we applied two different tests to confirm the positivity of HEV IgM and IgG results.

How and where our patient had been infected remains unclear; her case history contained no illuminating answers in this respect. She had no reported history of travelling to specific developing countries where HEV is a leading cause of acute viral hepatitis. Neither had she been exposed to potential zoonotic HEV transmission from, e.g., pigs in pig farms [28]. Also, because the observations were originally made in a clinical and occupational medicine context rather than in a research setting, neither molecular diagnosis (HEV RNA/RT-PCR) nor genotyping were possible. It remains therefore unclear by which viral genotype the student nurse had been infected.

\section{Conclusions}

In the discussed case, transient Anti-HAV IgM positivity, persistent Anti-HAV IgG positivity, HBsAg negativity, and persistent Anti-HBs positivity were due to hepatitis $A / B$ vaccination, while a coinciding HEV infection resulted in fatigue, high ALT and AST levels, and Anti-HEV IgM and IgG positivity.

Clinicians should appreciate that HEV infection can easily be overlooked in occupational medicine settings and in general practice. One should consider HEV infec- tion while dealing with each new hepatitis patient, especially because in a case as ours the originally observed characteristics were compatible with HAV infection in a hepatitis A vaccine non-responder. In such cases, renewed serological testing for HAV at a later stage, combined with HEV testing, is therefore worth considering.

\section{Conflict of Interest}

None declared.

\section{Source of Funding}

None declared.

\section{References}

1. Kamar N, Bendall R, Legrand-Abravanel F, Xia NS, ljaz S, et al. (2012) Hepatitis E. Lancet 379: 2477-2488.

2. Purdy MA, Smith DB, Simmonds P, Emerson SU, Harrison T, et al. (2014) New classification scheme for Hepeviridae. ICTV 1-15.

3. Smith DB, Simmonds P, International Committee on Taxonomy of Viruses Hepeviridae Study Group, Jameel S, Emerson SU, et al. (2014) Consensus proposals for classification of the family Hepeviridae. J Gen Virol 95: 2223-2232.

4. GeurtsvanKessel $\mathrm{CH}$, Islam Z, Mohammad QD, Jacobs BC, Endtz HP, et al. (2013) Hepatitis E and Guillain-Barré syndrome. Clinical Infectious Diseases 57: 1369-1370.

5. World Health Organization (2017) Hepatitis E.

6. De Schryver A, De Schrijver K, François G, Hambach R, van Sprundel M, et al. (2015) Hepatitis E virus infection: An emerging occupational risk? Occup Med (Lond) 65: 667-672.

7. Woo PC, Lau SK, Teng JL, Cao KY, Wernery U, et al. (2016) New hepatitis E virus genotype in Bactrian camels, Xinjiang, China, 2013. Emerg Infect Dis 22: 2219-2221.

8. Lee GH, Tan BH, Teo EC, Lim SG, Dan YY, et al. (2016) Chronic infection with camelid hepatitis $E$ virus in a liver transplant recipient who regularly consumes camel meat and milk. Gastroenterology 150: 355-357.e3.

9. Tabatabai J, Wenzel JJ, Soboletzki M, Flux C, Navid MH, et al. (2014) First case report of an acute hepatitis E subgenotype 3c infection during pregnancy in Germany. J Clin Virol 61: 170-172.

10. Aspinall EJ, Couturier E, Faber M, Said B, ljaz S, et al. (2017) Hepatitis E virus infection in Europe: surveillance and descriptive epidemiology of confirmed cases, 2005 to 2015. Euro Surveill 22.

11. European Centre for Disease Prevention and Control (ECDC) (2017) Hepatitis E in the EU/EEA, 2005-2015. Baseline assessment of testing, diagnosis, surveillance and epidemiology. SURVEILLANCE REPORT.

12. Suin V, Wautier M, Hutse V, Jacques $M$, Abady $M$, et al. (2017) Surveillance of hepatitis $E$ virus infection in Belgium: Epidemiological trends during the $2010-2016$ period. Scientific seminar on infectious diseases. WIV/ISP, Unit Epidemiology of Infectious Diseases 65.

13. Koot H, Hogema BM, Koot M, Molier M, Zaaijer HL (2015) Frequent hepatitis $\mathrm{E}$ in the Netherlands without traveling or immunosuppression. J Clin Virol 62: 38-40.

14. Kaba M, Moal V, Gerolami R, Colson P (2013) Epidemiology of mammalian hepatitis $E$ virus infection. Intervirology 56: $67-83$. 
15. Harvala $H$, Wong $V$, Simmonds $P$, Johannessen I, Ramalingam S (2014) Acute viral hepatitis-should the current screening strategy be modified? J Clin Virol 59: 184-187.

16. Bihl F, Negro F (2010) Hepatitis E virus: A zoonosis adapting to humans. J Antimicrob Chemother 65: 817-821.

17. Shouval D (2011) Focus. What is the significance of hepatitis $E$ as a public health threat in the industrialized countries? Hepatitis E virus infection in Italy. J Hepatol 54: 1-2.

18. EFSA Panel on Biological Hazards (BIOHAZ), Ricci A, Allende A, Bolton D, Chemaly M, et al. (2017) Public health risks associated with hepatitis $E$ virus (HEV) as a foodborne pathogen. EFSA Journal 15: e04886.

19. Mast EE, Weinbaum CM, Fiore AE, Alter MJ, Bell BP, et al. (2006) A comprehensive immunization strategy to eliminate transmission of hepatitis $B$ virus infection in the United States: Recommendations of the Advisory Committee on Immunization Practices (ACIP). Part II: Immunization of adults. MMWR Recommendations and Reports 55: 1-25.

20. Bonanni P, Bechini A, Pesavento G, Guadagno R, Santini MG, et al. (2006) Primary hepatitis A vaccination failure is a rare although possible event: Results of a retrospective study. Vaccine 24: 6053-6057.

21. Fiore AE, Wasley A, Bell BP (2006) Prevention of hepatitis A through active or passive immunization: Recommendations of the Advisory Committee on Immunization Practices (ACIP). MMWR Recommendations and Reports 55: 1-23.
22. Sjögren $\mathrm{MH}$, Hoke $\mathrm{CH}$, Binn LN, Eckels $\mathrm{KH}$, Dubois DR, et al. (1991) Immunogenicity of an inactivated hepatitis $A$ vaccine. Annals of Internal Medicine 114: 470-471.

23. Herrine SK (2017) Liver injury caused by drugs. In: Merck Manual. Professional Version.

24. Laine L, Goldkind L, Curtis SP, Connors LG, Yanqiong Z, et al. (2009) How common is diclofenac-associated liver injury? Analysis of 17,289 arthritis patients in a long-term prospective clinical trial. Am J Gastroenterol 104: 356-362.

25. Núñez M (2010) Clinical syndromes and consequences of antiretroviral-related hepatotoxicity. Hepatology 52: 11431155.

26. Giboney PT (2005) Mildly elevated liver transaminase levels in the asymptomatic patient. Am Fam Physician 71: 1105-1110.

27. Wenzel JJ, Preiss J, Schemmerer M, Huber B, Jilg W (2013) Test performance characteristics of anti-HEV IgG assays strongly influence hepatitis $E$ seroprevalence estimates. J Infect Dis 207: 497-500.

28. Geng J, Wang L, Wang X, Fu H, Bu Q, et al. (2011) Potential risk of zoonotic transmission from young swine to human: Seroepidemiological and genetic characterization of hepatitis $E$ virus in human and various animals in Beijing, China. J Viral Hepat 18: e583-e590. 